\title{
Telehealth care enhancement using the internet of things technology
}

\author{
Farouk Boumehrez ${ }^{1}$, A. Hakim Sahour ${ }^{2}$, Noureddine Doghmane ${ }^{3}$ \\ ${ }^{1,2}$ Department of Industrial Engineering, Faculty of Sciences and Technologies, ABBES Laghrour-Khenchela University, \\ Khenchela, Algeria \\ ${ }^{1}$ Laboratoire des Télécommunications, Faculty of Sciences and Technologies, 8 Mai 1945 University, Guelma, Algeria \\ ${ }^{2}$ Laboratoire systèmes et applications des technologies de l'information et des télécommunications (SATIT), Department \\ of Industrial Engineering, ABBES LaghrourUniversity, Khenchela, Algeria \\ ${ }^{3}$ Department of Electronics, Faculty of Engineering Sciences, BADJI Mokhtar University, Annaba, Algeria
}

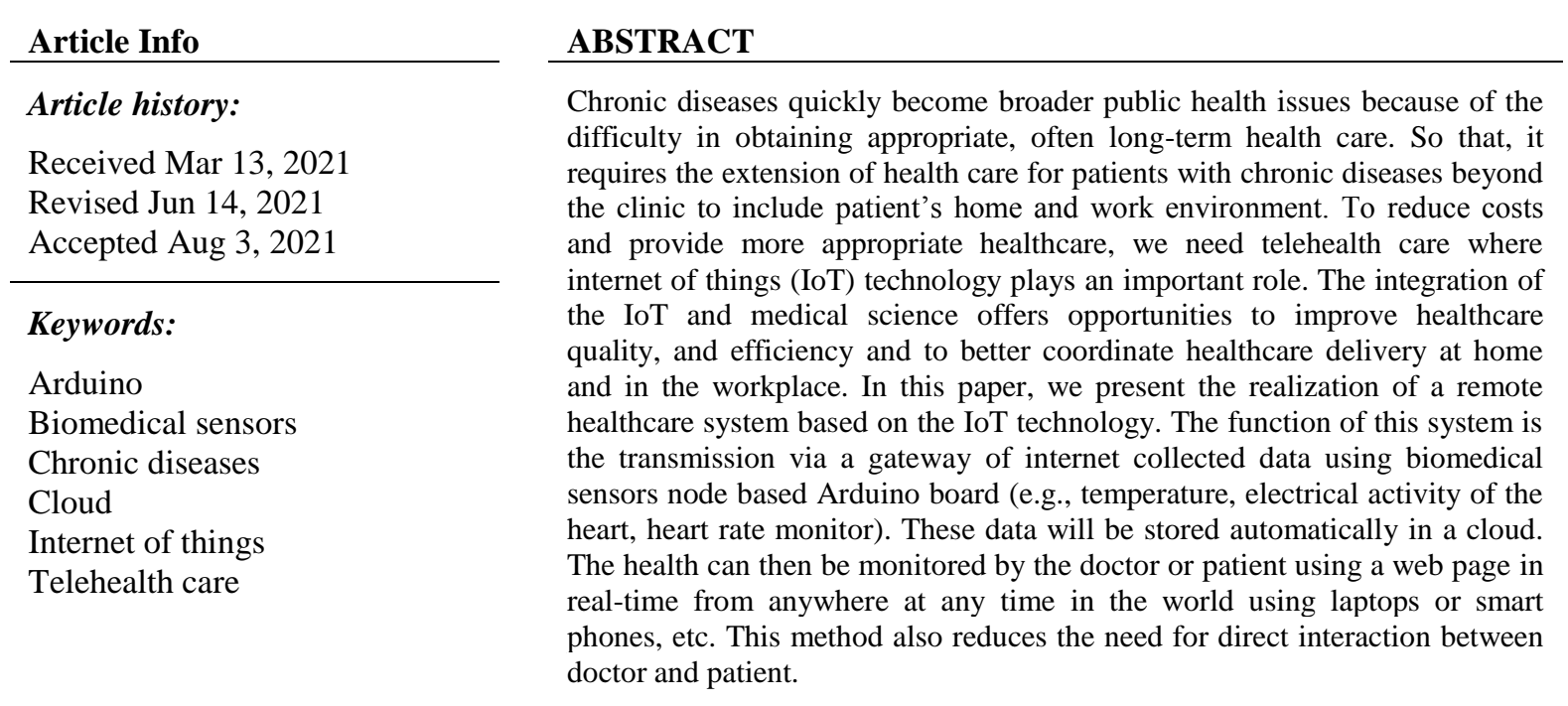

This is an open access article under the CC BY-SA license.

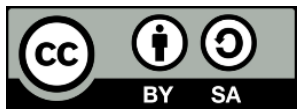

\section{Corresponding Author:}

Farouk Boumehrez

Department of Industrial Engineering

ABBES LaghrourUniversity

Khenchela 40004, Algeria

Email: boumehrez.farouk@univ-khenchela.dz

\section{INTRODUCTION}

The world population is aging. According to 2017 statistics from the World Health Organization, by 2050 , the number of people aged 65 years or over will account for more than 1.5 billion people, with most of the increase in developing countries [1], [2]. For example, in Canada, the number of people aged 65 or over is expected to increase from 4.8 million in 2010 to about 10.4 million in 2036 [3]. In addition, the number of people suffering from chronic diseases is increasing, which imposes restrictions on their physical condition. Chronic diseases disrupt the daily life of those who suffer from them and create financial, housing, employment or social problems. Moreover, social problems can occur with partners or children, on the sexual level, or in the practice of recreational activities [4]. Chronic diseases vary greatly in their symptoms, development, and treatment. Moreover, if not monitored and treated early, it can end the patient's life [5]. 
In many societies, health care providers and policy makers are interested in chronic diseases and consider them the cornerstone of modern health care [6], [7]. The United States of America spends annually $\$ 3.5$ trillion on healthcare, $90 \%$ of which is on caring for patients with chronic diseases [8]. This made the increasing demand on health care systems due to chronic diseases a major concern.

The circumstances of the COVID-19 pandemicthe world went through showed the necessity of introducing telemedicine procedures and providing medical services over the phone or through video calling. Which allows a constant flow of instantaneous data on patients' vital signs and relevant alerts, blended with information on lifestyle behaviors and social factors, and that'll make the health system will be better able to predict risks to patients [9], [10].

Telehealth includes telemedicine and other forms of data exchange about telehealth services. Telemedicine is a new form of medical practice that is now fully developed [11]. This allows the patients and doctors to be far away yet remain connected consistently with each other and get the required medical help on time. This is helpful for patients suffering from chronic illnesses or old age. It also helps the busy ones struggling with finding time to meet the physician in-person through the provision of out-of-hospital care [11], [12]. Information can be exchanged by email messages, two-way videos, smartphones, and other wireless gadgets. constant real-time health monitoring will provide substantial opportunities for personalizing people's healthcare experiences and interventions.

Medical internet of things (IoT) or IoT for healthcare is the use of advanced communication technologies and other related technologies, in the context of clinical health that provides care across distances [13]. IoT creates a comprehensive suite of IoT devices and applications exclusively designed for healthcare needs and settings [14], [15], such as sensors, remote healthcare monitoring applications, telemedicine consulting, etc. This type of monitoring is often used to manage high-risk patients, such as those with heart disease or people recently discharged from the hospital. Monitoring elderly patients at home or in living facilities can also be convenient and inexpensive [16], [17].

In this article, the IoT is used in the healthcare domain. The design proposed for our system meets the goal of the IoT, which is to enable things to be connected anytime, anyplace, with anything, and with anyone, ideally using any path or network and any service [17]. Moreover, our design is subject to client/server architecture and is based on the relationships that exist between the many applications in health monitoring [18]-[20]; i) gathering of data from sensors, ii) support of user interfaces and displays, iii) connectivity of networks and access to infrastructure services, iv) requirements of low power, robustness, durability, accuracy, and reliability. To identify the most appropriate vital parameters to reflect the human physical condition, many mobile emergency monitors and emergency case protocols have been examined [21]. Generally, many parameters are known to be important in human safety like body temperature, electrical activity of the heart (ECG), pulse and oxygen in blood, etc. as mentioned in [16], [22].

Various papers have explored the use of the IoT to control data in real-time. In [13] the authors focused on the utilization of IoT technology in the healthcare domain nowadays. This paper also presented an overview of the most important medical sensors in the IoT, as well as a review of the current state-of-the-art in IoT projects and technologies for healthcare services. Bhawiyuga, et al. [10] authors suggested the monitoring of telehealth by the IoT, they proposed the design of an IoT-cloud platform to incorporate both IoT devices and cloud entities by the use of Restful HTTP protocol as device-to-cloud data endpoint interface. Also, in [15], the researchers designed cloud-based middleware to enable the efficient web of things to access with batch and stream data to support medical wearable devices. On the other hand, in the study by [7] authors discussed mobile cloud computing in emergency healthcare model in case of an accident, this process includes an online preregister patient in the medical center to save the time of patient registration, this model allows the patients to review the previous feedback and experiences of each specialist or medical center and allows doctors to be able to stay in contact with their patients through mobiles applications.

In this paper, we propose a medical-IoT system to monitor and improve the life quality of patients in real-time on a 24/7 basis. This collected and concentrated data will affect normal mobile phone use. So, to make the control of data collection a reality without the patient inconvenience, the data is transferred directly to the cloud to be stored and processed effectively and efficiently. So that lets the authorized users access data in real-time from anywhere at any time. This reduces the need for direct interaction between doctor and patient.

\section{PROPOSED ARCHITECTURE}

In this paper, the system proposed allowing the measurement of many constants of the human body and will send them to the server where they will be stored, we've chosen body temperature, electrocardiogram, and pulse and oxygen in blood, but are not limited to, so that they can be increased, reduced, or even change depending on the patient's condition. 
In addition, the data presented on the Arduino board are then adjusted and transmitted in real-time, via a wireless connection (we used the Wi-Fi ESP-8266 module), to the cloud server. At that time, the data are permanently received by the server and saved in the database. This allows doctors or other users to view the data, in digital or graphical form, from a web browser on a device (smartphones, laptops, etc.) connected to the internet, allowing them to monitor vital signs and readings in real-time. Figure 1 shows the system structure. The system consists of two major parts; (1) an embedded system (patient); and (2) server application (cloud). The parts are explained in detail in the next section.

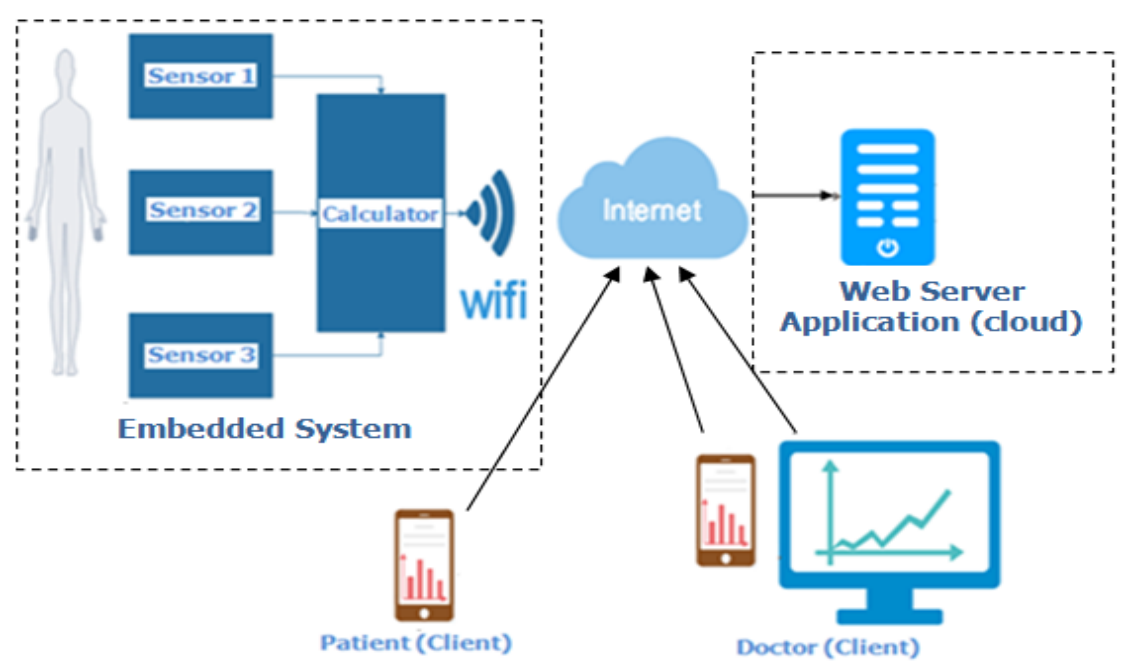

Figure 1. Block diagram of the proposed architecture

\subsection{Embedded system}

The embedded system in the form of a shield can be carried by the patients. Figure 2 shows the block diagram of the system. The design begins with the capture of patient health data from different sensors. These include body temperature sensors (LM335), electrocardiogram sensors (AD8232), and pulse and oxygen in blood sensors $\left(\mathrm{SaO}_{2}\right)$. In addition, the data presented on the Arduino board are then adjusted and re-send in real-time, via a wireless internet connection used the Wi-Fi ESP-8266 module, to the cloud server.

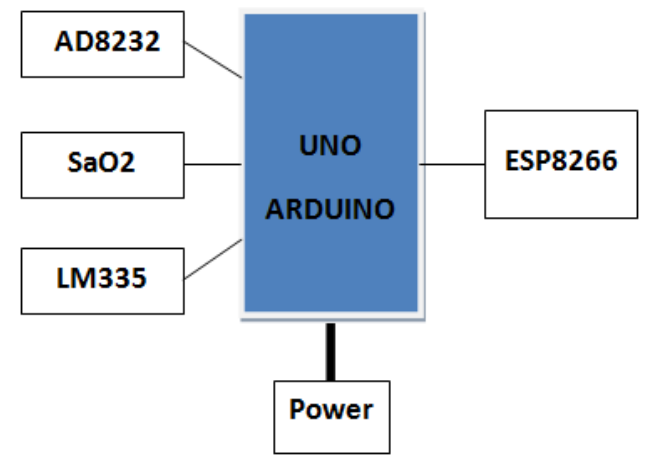

Figure 2. Embedded system of the remote monitoring system

\subsubsection{Arduino Uno}

Real-time embedded systems are generally used on the small end of the size scale [23]. For this purpose, the Arduino Uno microcontroller was used as the base station controller of the client part, we have opted for the Arduino Uno microcontroller board based on the ATmega328P, given that its characteristics are enough for the task [24]. It is inexpensive and compact; it eliminates the necessity of a computer and has low power consumption. 


\subsubsection{Sensor network part}

The input variables captured by network sensors. We opted for temperature measurement via an LM335 temperature sensor. For the ECG, an AD8232 heart rate monitor was used. The last parameter was the rate of oxygen saturation by a non-invasive sensor that is the photo plethysmo graph (PPG).

- Temperature sensor: the LM35 is a precision analog temperature sensor particularly suited for measuring temperatures below 150 degrees Celcius $\left({ }^{\circ} \mathrm{C}\right)$. It can be used for measurement of body temperature. It can be used for any type of temperature sensing in the range of $-40^{\circ} \mathrm{C}$ to $100^{\circ} \mathrm{C}$.

- The electrocardiogram sensor: the AD8232 electrocardiogram (ECG) is a cost-effective board used to assess the electrical and muscular functions of the heart. The AD8232 Single Lead Heart Rate Monitor acts as an operational amplifier to help easily obtain a clear signal from the PR and QT Intervals.

- Pulse and oxygen in blood sensor: the oxygen saturation $\left(\mathrm{SaO}_{2}\right)$ level in the blood is very important. Known as one of the "vitalsigns," it is one of the standard measurements taken by health professionals. $\mathrm{SaO}_{2}$ is defined as the percentage of hemoglobin with bound oxygen and is termed as $\mathrm{SpO}_{2}$ when measured by a pulse oximeter. The average level is 95-100 percent [22]. An oxygen level below 90 percent is considered to below, resulting in hypoxemia [25]. The noninvasive Pulse and Oxygen Sensor indicates the arterial oxygen saturation of functional hemoglobin. Oxygen saturation is defined as the measurement of the amount of oxygen dissolved in the blood, based on the detection of hemoglobin and deoxyhemoglobin.

\subsubsection{Wi-Fi module}

The famous ESP8266 Wi-Fi module, developed by Espressif, that permits any microcontroller integrated this circuit to access a wireless network. Moreover, it can communicate with the internet. It is a set of high performance, high integration wireless system on chips (SOCs) with an integrated TCP/IP protocol stack, constrained mobile platform designers, and uses $802.11 \mathrm{~b} / \mathrm{g} / \mathrm{n}$ protocols. It provides an unsurpassed ability to embed Wi-Fi capabilities within other systems or to function as a standalone application.

\subsection{Server application}

All data sent from the embedded system are permanently received by the server and saved in the database. This allows doctors or other users to view the data, in digital or graphical form, from a web browser on a device (smartphones, laptops, etc.) connected to the internet, allowing them to monitor vital signs and readings in real-time. In this part of the experiment, we used a computer that was configureured as a local server, on which we developed a routine to receive data from the Arduino board over the internet. The second one is used for decoding, recording, and processing data so that it can be exploited.

We opted for the XAMPP set [26], which is a free and open-source cross-platform web server solution stack package, extremely easy to install and use. The apache server comes bundled with MySQL database, PHP (a server side scripting language), Perl (another server side scripting language), the FileZilla FTP server, and the mercury mail transport system. If the doctor or patient wishes to get access to data sent from the Arduino part, he just needs to type the address of the website into an internet browser through laptops, smartphones, tablets, etc.

For this purpose, for each patient, we create a database that includes three tables (temperature, ECG, and pulse). An example is shown in Figure 3. MySQL database assigns sequence numbers automatically for each new value called the Index Column "ID". In table of temperature, ID presents the sequence numbers for recorded temperature values. The second column describes data from the temperature sensor. The following columns contain respectively the date and the time of receipt. In the same way, the ECG and the pulse sensors data are also recorded.

For any application, graphical user interfaces (GUIs) are key elements because they facilitate communication between humans and machines, as well as improve the performance of the application. Our interfaces were designed to meet a set of ergonomic requirements such as readability, comprehensibility, etc. Figure 4 presents a screenshot of the main application interface. It shows the set of interfaces that deal with real-time server data and displays data that are received digitally on a web page (body temperature, ECG, and blood pressure and oxygen).

Furthermore, it can be asserted that the graph form is a robust tool for data visualization [17]. It gives a genuine analysis and checks the healthcare of patients. Also, it can offer a high precise discussion and therefore a good decision making. It allows us to perceive the general pattern. For this purpose, we have provided a user convenient interface allowing him to access data that presented long-term historical parameters in graph form. 


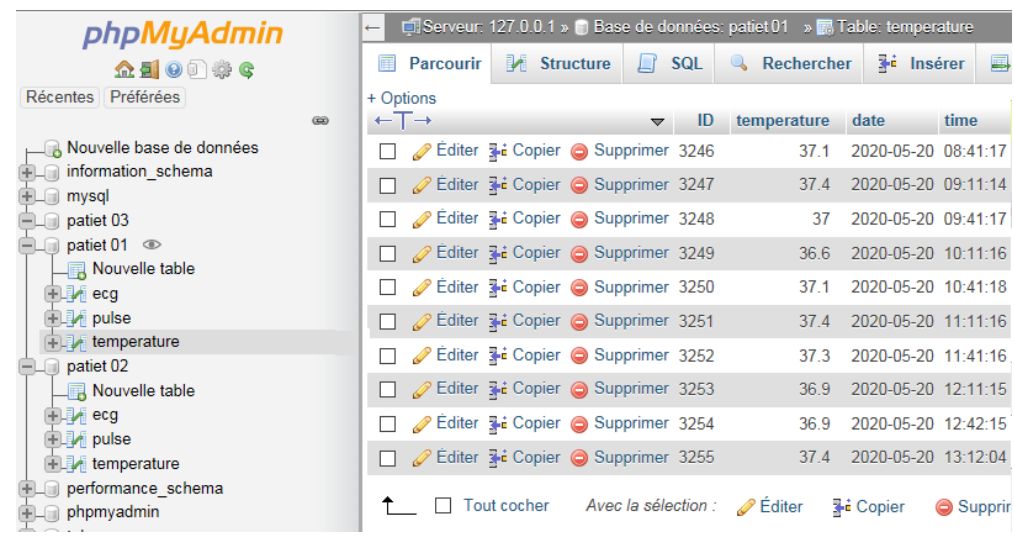

Figure 3. Database in MySQL

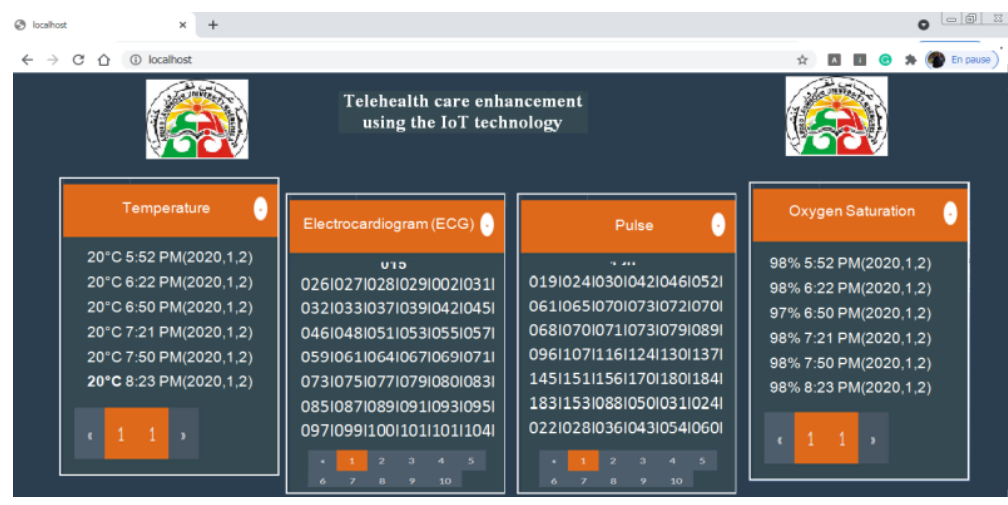

Figure 4. Screenshot of the main application interface

\section{RESULTS AND DISCUSSION}

In the healthcare domain, IoT is used to enhance the life quality of humans [10], which enables the healthcare system to monitor the associate patients. Also, The IoT helps people to perform basic tasks through an appropriate application. Indeed, this method gives better results in terms of the real-time monitoring of patients by attaching different sensors to measure body temperature, ECG, pulse, and oxygen in blood. Therefore, to improve treatment and responsiveness, the data collected by these sensors is made available on the internet to doctors, family members, and other interested parties. Aging and persons with disabilities who live alone that may require consistent supervision will find this device very helpful. In the final prototype for the designed system, all parts of ESP8266 Wi-Fi module and different sensors with Arduino Uno were assembled in Figure 5. In return, Figure 6 shows the hardware construction and testing.

Furthermore, additional experimental tests have been conducted to ensure the prototype functionality when applied to actual patients. In this experiment, samples were taken from university hospital patients and those who had no signs of any sickness from students and laboratory associates, i.e., the samples are a group of healthy and sick people of both sexes. Some of the participants are over 40 years old and with chronic illnesses. As well, for practical and fruitful monitoring, patients wear these wearable devices in both home environments and their workplaces. To validate the system, an experimental implementation was examined for long days in terms of sensor integration [13], in agreement with concerned users.

Moreover, Figure 7 shows an example of a pulse signal captured from the the Arduino serial plotter. This example presents how data is acquired by Arduino board from the pool sensor in graphical form. All acquired data are assembled and aranged in blocs, after they will be transmited via WIFI internet connection to the cloud. at this level they will be recevied and saved in database. Figure 8 demonstrates how the data is received by the server cloud. At the other end of the system, the supervising doctor or others can monitor the health of the patient via a set of devices (laptops, smartphones, or PCs) in conjunction with a web browser and an internet connection. The users can display received data in a digital or graph form. Figure 9 shows the ECG values which arrive consecutively on a single block according to the date and time of the received data. 
On the other hand, Figure 10 illustrates received data in graph form. Figure 10 (a) presents the graph of the variation of body temperature. Figure 10 (b) shows the ECG. Figure 10 (c) presents the pulse and in Figure 10 (d) we give the oxygen in blood according to time and date over the duration of the day for a particular user. Moreover, to prove the effectiveness of the proposed design, we compared the signals captured by the sensors and those displayed on web pages. As an example, when performing a comparison between the two Figures 7 and 10 (c), it can be seen that there is no significant difference between the two signals. This confirms the good performance of the proposed system.

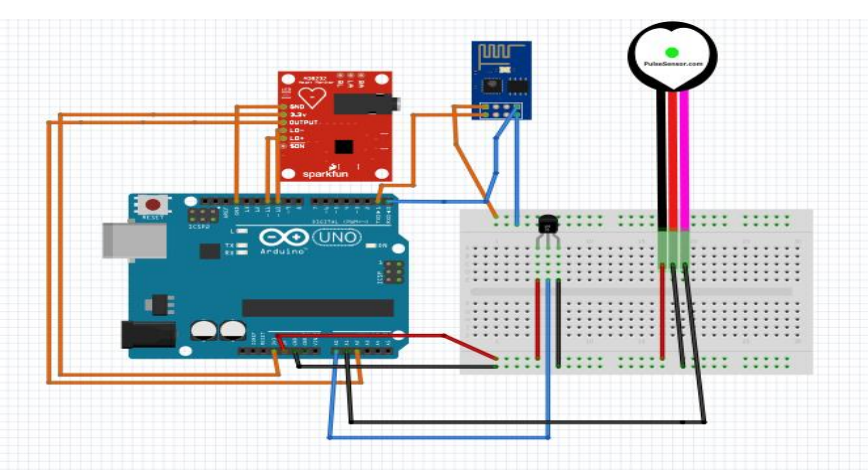

Figure 5. Interfacing of the ESP8266 wi-fi module and different sensors with Arduino Uno

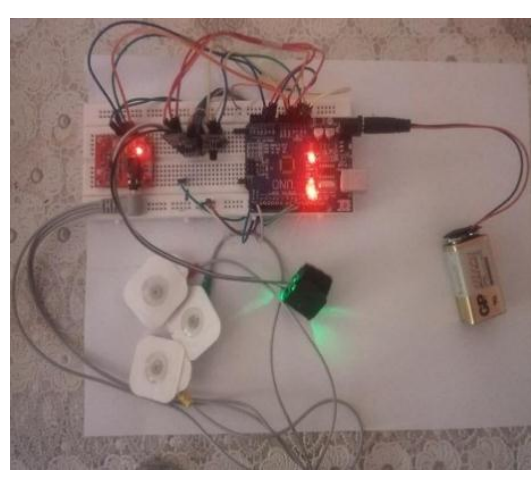

Figure 6. Hardware construction and testing

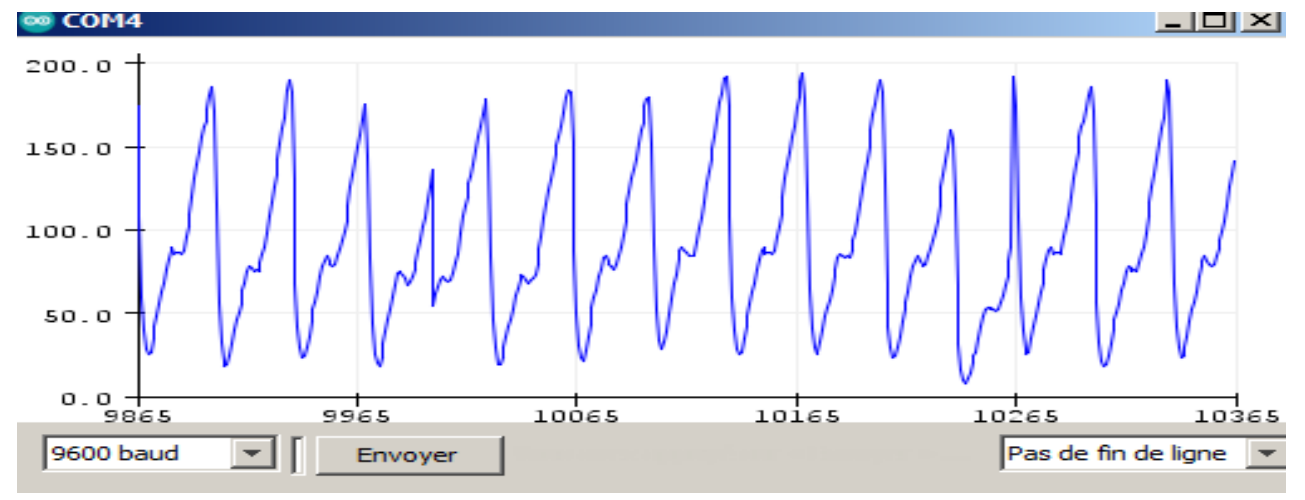

Figure 7. A pulse signal example captured from the Arduino serial plotter

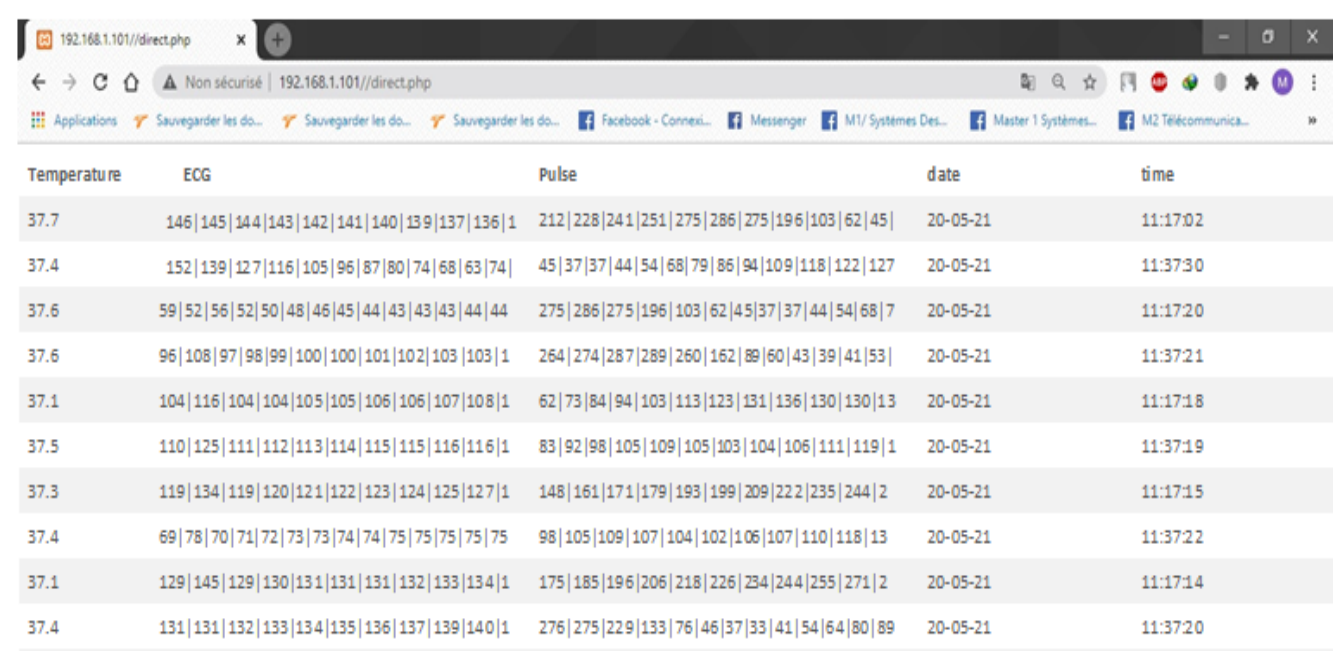

Figure 8. An example of the data received by the server 


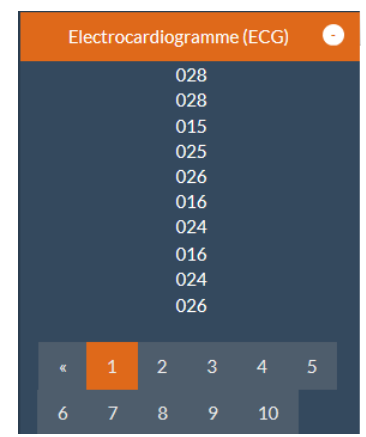

Figure 9. Received ECG data in digital form

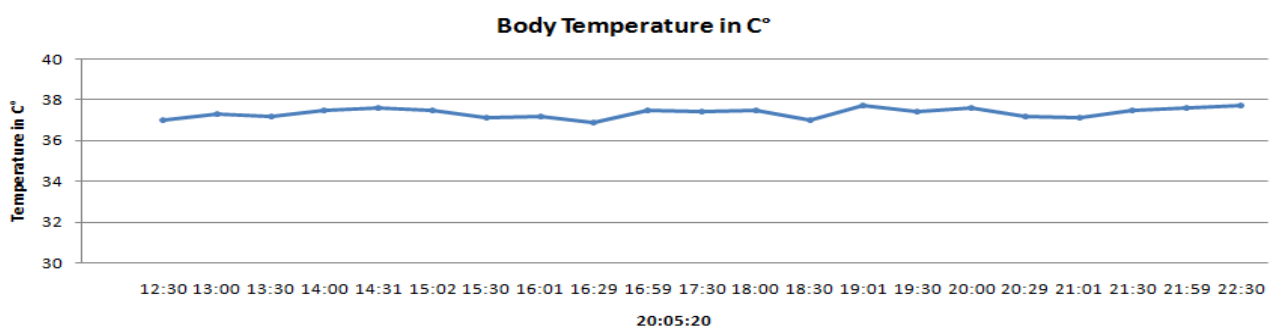

(a)

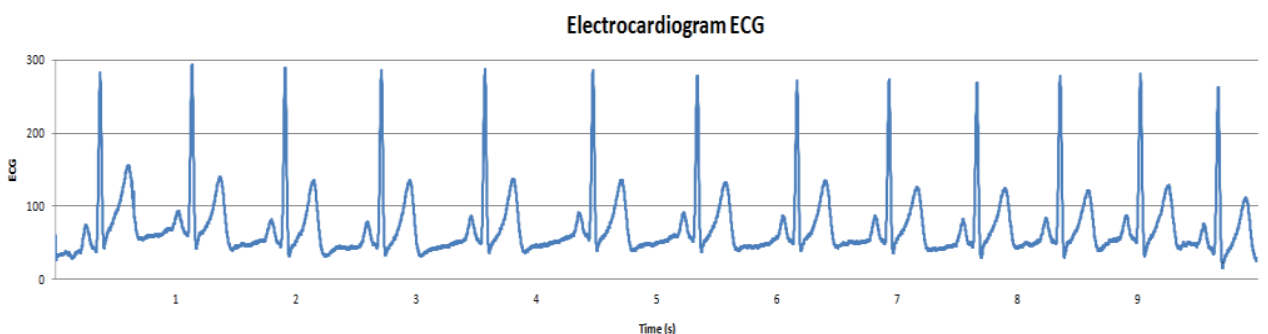

(b)

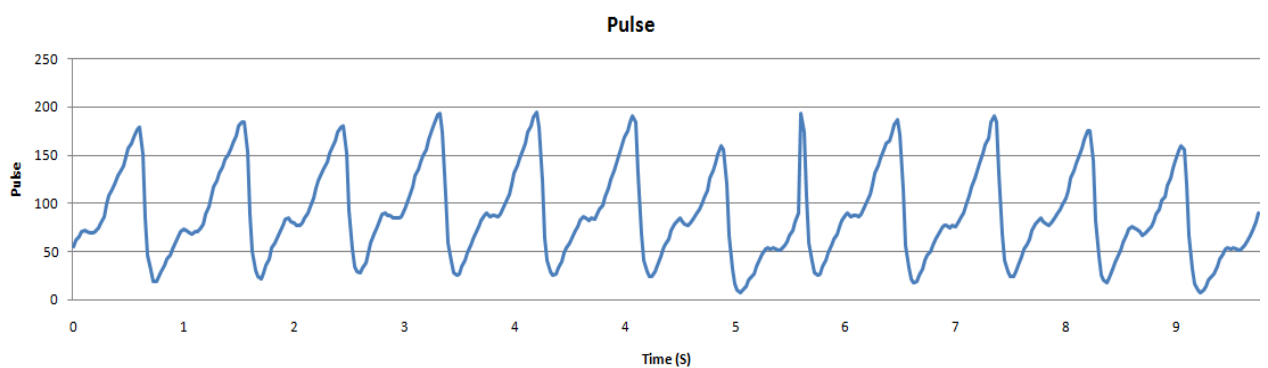

(c)

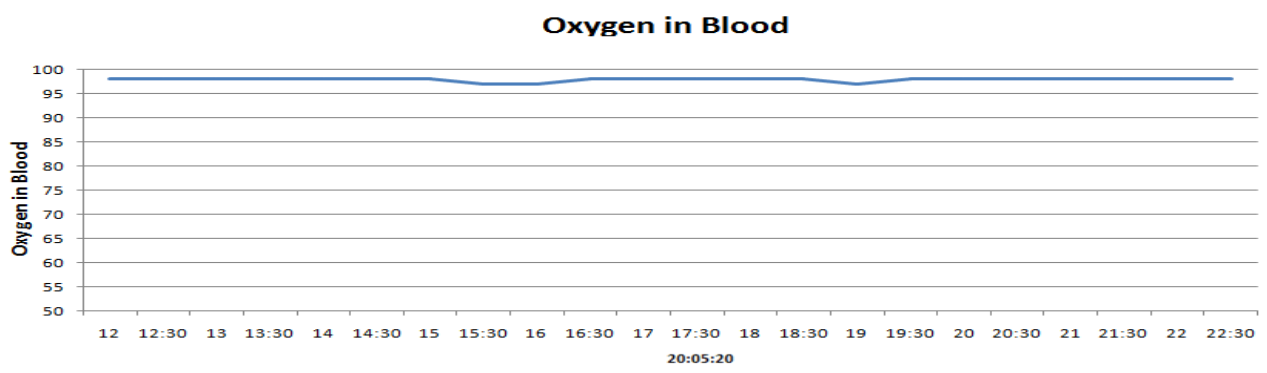

(d)

Figure 10. An example of data in graph form, (a) variation of body temperature, (b) ECG, (c) pulse, (d) oxygen in blood 


\section{CONCLUSIONS AND FUTURE WORK}

In this paper, a remote healthcare system based on the IoT technology method is proposed to improve the performance of healthcare quality, which could have been degraded at homes and work environments where Chronic disease control is strongly recommended. Moreover, a doctor can reach conclusions by examining and monitoring the health parameters of patients from remote locations. Any abnormal change in a patient's health parameters can alert the doctor and help him or her to take the necessary actions to save the patient's life.

Moreover, the patients, who contributed to the experiment, had positive feedback and give their gratitude to the system. Hence, this application gives much better results for monitoring users' healthcare as needed without the inconvenience of moving them to the hospital. So, they are satisfied with this precious service in their dailies. Clearly, this medical-IoT system has an enormous potential to improve healthcare delivery in both workplaces and home environments. The advantage of this work is an internet connection unit allows the system to be tracked in real-time by a web page in numerical or graph form. Hence, this method gives better results in terms of creating ideal healthcare for the patient through telemonitoring with normal life activities.

As future work, we are considering adding a set of signal processing procedures to allow data processing at the Arduino board level to reduce the flow of transmitted data. Also, we are developing procedures to help the server make decisions by itself and alert with SMSs. In addition, a backup system with a retransmission procedure in case of disconnection will be implemented. Moreover, the enormous amount of data recorded in the cloud requires using big data and cloud computing, which faster knowledge, analysis, and sharing of information.

\section{ACKNOWLEDGMENTS}

"This work was supported by the Directorate General for Scientific Research and Technological Development (DG-RSDT) of Algeria".

\section{REFERENCES}

[1] World Health Organization, "World report on ageing and health," Geneva, 2015. [Online]. Available: https://apps.who.int/iris/handle/10665/186463. [Accessed 20 December. 2020]

[2] H. Y. Yen, and L. J. Lin, "Quality of life in older adults: Benefits from the productive engagement in physical activity," Journal of Exercise Science \& Fitness, vol. 16, no. 2, pp. 49-54, 2018, doi: 10.1016/j.jesf.2018.06.001.

[3] M. Pirri, "Population aging and intergenerational equity in Canada," Public Policy and Governance Review, vol. 5, no. 2, pp. 28-38, Spring 2014.

[4] B. W. Ward, J. S. Schiller, and R. A. Goodman, "Multiple chronic conditions among US adults: A 2012 update," Preventing Chronic Disease., vol. 11, 2014, doi: 10.5888/pcd11.130389.

[5] M. Pasha, and S. M. W. Shah, "Framework for e-health systems in IoT-based environment," Wireless Communications and Mobile Computing, vol. 2018, pp. 1-11, 2018, doi: 10.1155/2018/6183732.

[6] V. Mach, M. Adamek, J. Sevcik, J. Valouch, and K. Barcova, "Design of an internet of things based real-time monitoring system for retired patients," Bulletin of Electrical Engineering and Informatics, vol. 10, no. 3, pp. 16481657, June 2021, doi: 10.11591/eei.v10i3.2699.

[7] S. A. Hameed, A. Nirabi, M. H. Habaebi, and A. Haddad "Application of mobile cloud computing in emergency healthcare," Bulletin of Electrical Engineering and Informatics, vol.8, no.3, pp. 1088-1095, September 2019, doi: 10.11591/eei.v8i3.1498.

[8] A. B. Martin, M. Hartman, D. Lassman, and A. Catlin, "National health care spending in 2019: Steady growth for the fourth consecutive year external icon," Health Aff, vol. 40, no. 1, pp. 1-11, 2020, doi: 10.1377/hlthaff.2020.02022.

[9] R. Galiero et al., "The importance of telemedicine during COVID-19 Pandemic: A focus on Diabetic Retinopathy," Journal of Diabetes Research, vol. 2020, pp. 1-8, 2020, doi: 10.1155/2020/9036847.

[10] A. Bhawiyuga, D. P. Kartikasari, K. Amron, O. B. Pratama, and M. W. Habibi, "Architectural design of IoT-cloud computing integration platform," Telkomnika (Telecommunication, Computing, Electronics and Control), vol. 17, no. 3, pp. 1399-1408, 2019, doi: 10.12928/telkomnika.v17i3.11786.

[11] S. Tennina et al., "WSN4QoL: WSNs for remote patient monitoring in e-Health applications," 2016 IEEE International Conference on Communications (ICC)., Kuala Lumpur, pp. 1-6, 2016, doi: 10.1109/ICC.2016.7511597.

[12] S. L. Gorst, C. J. Armitage, S. Brownsell, and M. S. Hawley "Home telehealth uptake and continued use among heart failure and chronic obstructive pulmonary disease patients: A systematic review," Ann Behav Med., vol. 48, no. 3, pp. 323-336, 2014, doi: 10.1007/s12160-014-9607-x.

[13] N. M. M. AbdElnapi, N. F. cvioOmran, A. A. Ali, and F. A. Omara, "A survey of internet of things technologies and projects for healthcare services," 2018 International Conference on Innovative Trends in Computer Engineering (ITCE), Aswan, 2018, pp. 48-55, doi: 10.1109/ITCE.2018.8316599. 
[14] A. Rhayem, M. B. A. Mhiri, M. B. Salah, and F. Gargouri, "Ontology-based system for patient monitoring with connected objects," Procedia Computer Science., vol. 112, pp. 683-692, 2017, doi: 10.1016/j.procs.2017.08.127.

[15] A. Bhawiyuga, S. A. Kharisma, B. J. Santoso, D. P. Kartikasari, and A. P. Kirana "Cloud-based middleware for supporting batch and stream access over smart healthcare wearable device," Bulletin of Electrical Engineering and Informatics, vol. 9, no. 5, pp. 1990-1997, October 2020, doi: 10.11591/eei.v9i5.1978.

[16] D. Dias, and J. P. S. Cunha, "Wearable health devices-vital sign monitoring, systems and technolgogies," Sensors, vol. 18 , no. 8, 2018, doi: 10.3390/s18082414.

[17] A. M. Johansson, I. Lindberg, and S. Söderberg, "Healthcare personnel's experiences using video consultation in primary healthcare in rural areas," Primary Health Care Research \& Development, vol. 18, no. 1, pp. 73-83, 2017, doi: $10.1017 / \mathrm{S} 1463423616000347$.

[18] V. Ovidiu, et al., "Internet of things strategic research and innovation agenda," In: Ovidiu Vermesan, Peter Friess, editors. Internet of Things Applications-From Research and Innovation to Market Deployment. Aalborg (Denmark): River Publisher. pp. 7-142, 2014. JRC90851 [Online]. Available: http://www.internet-of-thingsresearch.eu/pdf/IERC_Cluster_Book_2014_Ch.3_SRIA_WEB.pdf. [Accessed 01 January 2021].

[19] V. Tikhvinskiy, G. Bochechka, A. Gryazev, and A. Aitmagambetov, "Comparative analysis of QoS management and technical requirements in 3GPP standards for cellular IoT technologies," Journal of Telecommunications and Information Technology. vol. 2, pp. 41-47, 2018, doi: 10.26636/jtit.2018.122717.

[20] B. Saneja, and R. Rani, "An efficient approach for outlier detection in big sensor data of healthcare," Int J Commun Syst., e3352, pp. 1-10. 2017, doi:10.1002/dac.3352.

[21] F. N. Shuhaimi, N. Jamil, and R. Hamzah, "Evaluations of Internet of Things-based personal smart farming system for residential apartments," Bulletin of Electrical Engineering and Informatics, vol. 9, no. 6, pp. 2477-2483, December 2020, doi: 10.11591/eei.v9i6.2496.

[22] Z. J. V. Cohen, S. Haxha, and A. Aggoun, "Pulse oximetry optical sensor using oxygen-bound haemoglobin," Optics Express., vol. 24, no. 9, pp. 10115-10131, 2016, doi: 10.1364/OE.24.010115.

[23] H. Jahanirad, and H. Karam, "BIST-based testing and diagnosis of LUTs in SRAM-based FPGAs," Emerging Science Journal., vol. 1, no. 4, pp. 216-255, 2017, doi: 10.28991/ijse-01125.

[24] A. Sahour, F. Boumehrez, and M. Benouaret, "Greenhouse climate controller by using of internet of things technology and fuzzy logic," Instrumentation Mesure Métrologie, vol. 20, no. 1, pp. 29-38, February 2021, doi: 10.18280/i2m.200105.

[25] L.Wang, and R. Jones, "Big data analytics for disparate data," American Journal of Intelligent Systems., vol. 7, no. 2, pp. 39-46, 2017, doi: 10.5923/j.ajis.20170702.01.

[26] S. Hussain, Z. Wang, S. Rahim, "E-learning Services for Rural Communities," International Journal of Computer Applications, vol. 68, no. 5, pp. 15-20, 2013, doi: 10.5120/11574-6888. 